\title{
Treatment of Non-erosive Effect of Clinical Study of Gastroesophageal Reflux Disease
}

\author{
Liu Qian \\ Heze City, Shandong Province, China Hospital of Heze, Shandong Province, 274035, China.
}

Abstract: Objective: To observe the clinical effect of "Chaihu Shugan Powder Zuojin Pill" in the treatment of non-erosive gastroesophageal reflux disease. Methods: Sixty patients with non-erosive gastroesophageal reflux disease were randomly divided into treatment group and control group (30 cases in each group). The patients were treated with "Zaohu Shugan Powder" and "Zangjin Pills" and omeprazole respectively. For 8 weeks, the clinical efficacy and changes in clinical symptoms were observed. Results: The total effective rate was $93.3 \%$ in the treatment group and $80.0 \%$ in the control group. There was significant difference between the two groups before and after treatment $(\mathrm{P}$ $<0.05)$. Conclusion: "Chaihu Shugan Powder Zuojin Pill" can effectively improve the clinical symptoms of non-erosive gastroesophageal reflux disease.

Keywords: "Chaihu Shugan Powder Zuojin Pill"; non-erosive reflux disease

\section{Introduction}

Gastroesophageal reflux disease (GRD) refers to a disease in which the contents of the stomach are refluxed into the esophagus, causing discomfort and/or complications. GRD can be divided into non-erosive reflux disease (NERD), erosive esophagitis and Barrett's esophagus. In the three types of GRD, NERD accounted for about $70 \%$. In 2007, Switzerland Vevey International Conference reported that NERD is a subtype of GRD, which is a reflux-related symptom. In the near future, without receiving acid therapy in the case of routine endoscopy failed to find esophageal mucosal erosion or interruption of the disease ${ }^{[1]}$. This study aims to find the effects of "Chaihu Shugan Powder Zuojin Pill" and omeprazole in the treatment of non-erosive GRD.

\section{Clinical data}

\subsection{General information}

All the cases studied in this study were outpatient and inpatient cases in our hospital, and 60 cases were included in the standard. There were 8 males and 23 females with an average age of $53.23 \pm 14.33$ years. There were 9 males and 22 females with an average age of $54.90 \pm 11.53$ years. The sex and age of the two groups were comparable $(\mathrm{P}>0.05)$.

Copyright (C) 2017 Liu Q.

doi: 10.18686/aem.v6.89

This is an Open Access article distributed under the terms of the Creative Commons Attribution-Non Commercial 4.0 International License (http://creativecommons.org/ licenses/by-nc/4.0/), permitting all non-commercial use, distribution, and reproduction in any medium, provided the original work is properly cited. 


\subsection{Diagnostic criteria}

Diagnostic criteria of the non-erosive GRD is based on "Chinese Gastroesophageal Reflux disease consensus"[2].(1) Typical symptoms include pantothenic acid, heartburn, sternal pain discomfort, and nausea.(2)Electronic gastroscopy: Patient with esophageal mucosal erosion was not included in the endoscopic examination.Besides, patients with Barrett's esophagus, erosive esophagitis and complications, such as esophageal inflammation stenosis, ulcers, and esophageal cancer were excluded.

\subsection{Inclusion criteria}

(1) Age 18-65 years of age, gender is not limited; (2) there is a typical reflux symptoms at least for more than 3 months; (3) reflux disease questionnaire (RDQ) $\geq 12$ points; (4) no mucosal damage showed in electronic endoscopy; (5) signed informed consent.

\subsection{Exclusion criteria}

(1) Electronic gastroscopy examination showed esophageal mucosal erosion, ulcers, Barrett's esophagus, stomach, esophageal or duodenal surgery history, peptic ulcer, upper gastrointestinal malignant lesions, dugouts esophagitis, and pyloric obstruction ; (2) liver and kidney dysfunction, severe cardiovascular disease; (3) pregnant and lactating women; (4) non-collaborators (that cannot be diagnosed with endoscopy or cannot be prescribed medication and affect the efficacy);(5) patients with mental illness and who cannot communicate with the doctor.

\section{Treatment and observation methods}

\subsection{General treatment}

Patients were forbidden to eat spicy, greasy and other spicy food. Besides, patients should avoid too full and food consuming $2 \mathrm{~h}$ before going to bed. The bed should be raised to $15-20 \mathrm{~cm}$. Patient should quit smoking and alcohol. Patient should avoid food intake that decrease lower esophageal sphincter (LES), such as chocolate, tea, high-fat foods and high-acid foods.

\subsection{Drug treatment}

Treatment group was given "Chaihu Shugan Powder Zuojin Pill", from bupleurum, Citrus aurantium, dried tangerine peel, Cyperus rotundus, Chuanxiong, white peony root, berberine, and evodia, 1 dose daily and split to 2 times orally. If epigastric swelling, add magnolia back; if sternum showed obvious pain, add corydalis tuber and fructus meliae toosendan; if nausea, add Rhizoma Pinelliae Preparata and Amomum Villosum. The control group received omeprazole $20 \mathrm{mg} /$ tablets, $20 \mathrm{mg}, 2$ times a day, and half an hour before meals orally. Treatment was given for 8 weeks. An evaluation was carried out after the end of treatment.

\subsection{Observation method}

The RDQ score of 40 points includes the sum of the frequency and degree of symptoms. Symptoms include acid reflux, heartburn, chest pain, and nausea. The four symptoms are recorded according to the severity and frequency. The frequency of the onset of four major clinical symptoms of patients was recorded as: never been, 1 week $<1 \mathrm{~d}, 1$ week $1 \mathrm{~d}$, 1 week 2-3d, 1 week 4-5 days, and almost every day. The points are calculated statistically as $0,1,2,3$, 4, and 5 points. The degree of symptoms of patients were divided into six grades: no, very mild, mild, moderate, severe and very severe, as $0,1,2,3,4$ and 5 score, respectively. 


\subsection{Statistical methods}

SPSS 20.0 statistical software was used for statistical analysis. Group data were compared using t-test. The measurement data were expressed as mean \pm standard deviation $(\bar{x} \pm \mathrm{s})$. The counting data were compared by using chi-square test.

\section{Observation of efficacy}

\subsection{Criteria for the determination of efficacy ${ }^{[3]}$}

Recovery: clinical symptoms disappeared or disappeared, the score decreased $\geq 95 \%$;

Markedly: symptoms and signs improved significantly, points decreased by $\geq 70 \%$ and $<95 \%$;

Effective: symptoms and signs are improved, points reduction $\geq 30 \%$ and $<70 \%$;

Invalid: symptoms and signs were no significant improvement or increase, points decreased by $<30 \%$.

\subsection{Results}

\subsubsection{Comparison of clinical efficacy}

The total effective rate of the treatment group was $93.3 \%$. The total effective rate of the control group was $80.0 \%$. The difference between the two groups was statistically significant $(\mathrm{P}<0.05)$. Refer to Table 1 .

Table 1 Comparison of clinical efficacy between two groups

\begin{tabular}{lllllll}
\hline Group & No. of cases & Recover & Efficiency & Effective & Ineffective & Total efficiency (\%) \\
\hline Treatment group & 30 & 3 & 22 & 3 & 2 & $93.3^{*}$ \\
Control group & 30 & 1 & 16 & 7 & 6 & 80.0 \\
\hline
\end{tabular}

Note: $* \mathrm{P}<0.05$ when compared with the control group after treatment.

\subsubsection{Comparison of symptom scores}

There was no significant difference between the two groups before treatment $(\mathrm{P}>0.05)$. There was significant difference between the two groups before and after treatment $(\mathrm{P}<(\mathrm{P}<0.05)$. The difference between the two groups was statistically significant $(\mathrm{P}<0.05)$. Refer to Table 2 .

\section{Discussion}

GRD is not presence in the record of traditional Chinese medicine. The majority of doctors determine the disease according to the clinical manifestations. The pathogenesis of NERD is related to the positions in the esophagus, stomach, liver and spleen ${ }^{[4]}$. As the patient diet section and other direct damage to the spleen and stomach, and affecting the qi. 
Table 2 Symptoms of two groups before and after treatment $\left(\bar{x}_{ \pm \mathrm{s}}\right)$.

\begin{tabular}{lll}
\hline Group & Before Treatment & After Treatment \\
\hline Treatment group & $18.90 \pm 0.26$ & $4.17 \pm 0.18^{\mathbf{*}}$ \\
Control group & $19.73 \pm 0.24$ & $9.03 \pm 0.19^{-}$ \\
\hline
\end{tabular}

Note: $\square \mathrm{P}<0.05$ when compared with the control group before treatment; $\circ \mathrm{P}<0.05$ when compared with the control group after treatment.

The Bupleurum is slightly bitter. The "Ben-Jing" records: "the stomach accumulates gas, food accumulation, cold and heat, pushing the old away"; "Materia Medica by 100 kinds record" contains: "Bupleurum is drug for the stomach, the smell is light and fresh, remove stagnation of qi."

Modern pharmacology study proved that Bupleurum, Cyperus rotundus, Citrus aurantium can promote smooth muscle contraction, increase the tension of the pyloric sphincter, relaxation Oddi sphincter, promote gastrointestinal emptying, and thereby relieve esophageal gastric motility disorders. Bupleurum in the total Saponins and Citrus Aurantium volatile oil can significantly reduce the secretion of gastric juice, reduce the activity of pepsin, and improve the $\mathrm{pH}$ value of gastric juice ${ }^{[5]}$. Coptis, Evodia and licorice can inhibit gastric acid secretion and reduce the pepsin activity $^{[6]}$. Total use of drugs can reduce or even inhibit gastric acid secretion, inhibit pepsin activity, increase gastrointestinal motility, enhance gastrointestinal emptying, relieve or restore esophageal and gastrointestinal motility disorders.

In conclusion, "Chaihu Shugan Powder Zuojin Pill" is effective for treatment of non-erosive GRD and it is worthy of clinical use.

\section{Reference}

1. Vakil N., van Zanten SV., Kahrilas P., et al. The Montreal definition and classification of gastroesophageal disease: A global evidence-based consensus [J]. Am J Gastroenterol, 2006,101 (8): 1900-1920.

2. SanRen Lin, GuoMing Xu, PinJin $\mathrm{Hu}$, et al. Chinese gastroesophageal reflux disease consensus opinion [J]. Gastroenterology, 2007, 12 (4): 233-299.

3. XiaoYu Zheng. Traditional Chinese medicine clinical research guidelines [M]. Beijing: China Medical Science and Technology Press, 2002: 215-220.

4. ShengLiang Zhu, YongShun Sun, ShuPing Ma. Gastroesophageal reflux disease traditional Chinese medicine syndrome analysis [J]. Shanghai Journal of Traditional Chinese Medicine, 2006,40 (10): 28-29.

5. QianZheng Li. Traditional Chinese medicine of gastroenterology [M]. Beijing: China Medical Science and Technology Press, 1993: 446.

6. FuQiang Ye, WeeWen Chen. Pharmacological research of “ZuoJinWan” [J]. Chinese Patent Medicine. 1998, 18 (1): 43. 